COMPTES RENDUS

\title{
SCIENCES SOCIALES ET HISTOIRE
}

Jean-Claude HocQuet, Le Sel et le pouvoir. De l'An mil à la Révolution française. Paris, Albin Michel, 1984. 13,5 × 21, 517 p., index, bibliogr. ("L'aventure humaine $"$ ).

Aucun livre de J.-C. Hocquet ne laisse indifférent. Celui-ci n'échappe pas à la règle. Deux préoccupations majeures semblent s'y entrecroiser ; d'une part, élaborer une somme sur l'histoire européenne du sel à l'ouest d'une ligne LübeckVenise "de l'An mil à la Révolution française"; de l'autre, contribuer ainsi à l'histoire plus générale du féodalisme, en s'appuyant sur l'étude d'un produit clé, bien plus indispensable alors que de nos jours. Le premier objectif s'affirme dans l'introduction; le second se dévoile peu à peu et s'impose dans la conclusion.

La première ambition est pleinement satisfaite. J.-C. Hocquet met à la disposition du chercheur français une masse considérable d'informations et, qui plus est, minutieusement et comme à son habitude, solidement critiquées (en particulier les données chiffrées). De cette accumulation de faits, il essaie, en outre, de tirer une vue d'ensemble qui insiste également sur les transformations progressives et sur les ruptures décisives. Son mérite est grand car, selon les siècles, les travaux, souvent ponctuels, portent plutôt sur la production, plutôt sur le commerce ou plutôt sur la fiscalisation. De tous ces efforts, il résulte une synthèse tout à la fois utile et originale, employant et dominant au mieux les acquis actuels.

Le second projet s'intègre aux tentatives contemporaines d'historiens marxistes qui cherchent à définir et à préciser le concept de féodalisme. La position adoptée par J.-C. Hocquet se situe tout à la fois dans la ligne de ses prédécesseurs, et s'en écarte aussi notablement. Elle s'y situe en ce que l'auteur insiste sur une transformation du féodalisme au moins à travers une crise (placée au $\mathrm{XIII}^{\mathrm{e}}$ siècle, donc nettement avant ce qu'a proposé Guy Bois). Elle s'en écarte, car elle ne voit pas dans le grand commerce une forme du capitalisme ou une forme précurseur du capitalisme, mais un phénomène "féodaliste ", c'est-à-dire parfaitement intégré au féodalisme. En outre, le non-marxiste se demande où J.-C. Hocquet voit la "contradiction fondamentale " du système féodal. Du

Revue de synthèse: IV S. Nos 3-4, juil.-dèc. 1988. 
moins n'apparait-elle pas clairement au profane qui n'est pas initié à toutes les arcanes et à toutes les polémiques de l'école.

Peut-être rejoint-on là la seule réserve sérieuse que l'on puisse faire à l'ouvrage en dehors des discussions sur l'existence ou la nature du féodalisme. Les raisonnements de J.-C. Hocquet demeurent beaucoup trop souvent implicites et le lecteur perd facilement la démarche de l'auteur, parce que celui-ci ne lui livre pas toujours les repères nécessaires. C'est dommage car il a pris ainsi le risque de décourager ou au moins d'irriter ce lecteur. Aussi conseillerons-nous à ce dernier de surmonter sa lassitude ou son agacement et de poursuivre.

Hugues NeveuX.

Jean PITIÉ, L'Homme et son espace : l'exode rural en France du xVf siècle à nos jours. Paris, C.N.R.S., 1987. $16 \times 24,662$ p.

Cette nouvelle édition actualisée rend accessible un outil de travail essentiel. Jean Pitié a, en effet, rassemblé tous les ouvrages traitant de l'exode rural, dans un ordre chronologique du $\mathrm{Xvi}^{\mathrm{e}}$ siècle à 1987 (p. 41 à 240) puis régional (p. 245 à 638) (DOM-TOM compris). Chaque livre ainsi répertorié est rapidement analysé. Cette magistrale bibliographie est complétée par des cartes, des statistiques, des textes choisis et des index qui font de ce volume le point de départ obligé de toute recherche sur le sujet. Le bel ouvrage tient ici à l'ampleur de la tâche conjuguée à l'humilité du traitement. A son utilisation pour la recherche, on pourra ajouter le simple plaisir d'une promenade presque poétique à travers des centaines de titres et des centaines de lieux.

Pierre MONZANI.

Steven Laurence Kaplan, Provisioning Paris. Merchants and Millers in the Grain and Flour Trade during the Eighteenth Century. Ithaca/Londres, Cornell University Press, $1984.16 \times 24,5,666$ p., bibliogr., index, ill.

Par bien de ses aspects, par nombre de ses caractères, ce gros livre du professeur Kaplan' s'inscrit en fait dans la postérité des travaux de Jean Meuvret; il en parait la continuation au meilleur sens du terme. Comme eux, il s'intéresse au "problème des subsistances ", ici à l'approvisionnement de la capitale. A leur instar, il s'appuie sur de vastes dépouillements d'archives et une grande maitrise de la litterature contemporaine, périodiques, essais, traités. Il en a enfin' la précision, la minutie et en fin de compte la solidité ; c'est-à-dire

1. Traduction française : Les Ventres de Paris. Pouvoir et approvisionnement dans la France d'Ancien Régime, Paris, Fayard, 1988. 
qu'il révèle une méfiance des grands mots au contenu flou, qui facilitent les glissements de sens insensibles et inconscients.

Pourtant, l'ouvrage de Steven L. Kaplan se différencie aussi nettement des articles de Jean Meuvret. Fondamental sans doute est ici le décalage chronologique, le "centrage" sur le xviII siècle. Car si dans le domaine de l'agronomie et du "ménage des champs", Jean Meuvret s'était attentivement penché sur ce siècle, son intérêt en ce qui concerne le ravitaillement de Paris, s'était plutôt concentrè sur " l'époque Louis XIV ». Il résulte de ce déplacement que Steven L. Kaplan met bien en relief les transformations qui surviennent au siècle des Lumières : déclin du commerce de Grève (et des négociants de la rue de la Mortellerie), montée en puissance du commerce de la farine qui surpasse à partir de la décennie 1730 celui des grains, importance grandissante dans le sillage de ce glissement des boulangers, puis des marchands-meuniers qui en sont les initiateurs (et les bénéficiaires), développement mal endigué par les autorités de la livraison "en droiture", enfin, en raison du poids désormais déterminant de la farine, intérêt croissant porté aux techniques de la meunerie que symbolise l'essor de la "mouture économique".

Comme l'avait suggéré Jean Meuvret, Steven L. Kaplan reconnaît la formation de réseaux familiaux qui autorisent des informations sûres, des règles d'échange respectées, une confiance mutuelle. Mais il insiste beaucoup aussi sur le revers de cette organisation : la fragilité de ces réseaux, leur incapacité à promouvoir un négoce de très large envergure, la faiblesse d'un crédit aux bases trop serrées pour encaisser correctement les à-coups un peu violents.

Il y a enfin chez Steven $L$. Kaplan une vue synthétique née de la précision et de la minutie de son étude et qui, quoiqu'elle soit exprimée dès les premiers chapitres, en constitue en définitive l'unité. Il estime, en effet, après d'autres certes, dont Jean Meuvret, bien que peut-être plus systématiquement qu'eux, que le commerce des grains et de la farine n'est pas, à l'époque, un négoce comme les autres, qu'il relève en réalité de la "bonne police" d'un "bon gouvernement " qui se doit de le réglementer faute d'être capable de l'assumer lui-même. Dans cette perspective, les intermèdes «libéraux " de 1763-1770 et de 1774-1776 fournissent la contre-épreuve recherchée.

$\mathrm{Au}$ terme de la lecture, on peut seulement regretter qu'en conclusion, l'auteur, au lieu de résumer le contenu de son livre, n'ait pas justement tenté de déterminer dans quelle mesure les transformations qu'il a enregistrées contribuèrent à ébranler le système, même s'il se risque à èmettre l'hypothèse qu'au XIX siècle les "attitudes" anciennes aient survécu à la lente et progressive évolution des "pratiques ". Mais qui, devant la richesse et l'honnêteté de son travail, oserait le lui reprocher?

Hugues NeVEUX. 
La Ville et l'innovation : relais et réseaux de diffusion en Europe, XIV-XIX siècle. Dir. Jochen Hoock, Bernard LEPETIr. Paris, Ed. de l'E.H.E.S.S., 1987. $15 \times 23,220$ p., graph., tabl., cartes ( « Recherches d'histoire et de sciences sociales ", 23).

L'historien des villes et des circulations socioculturelles trouvera matière à réflexion et perspectives méthodologiques stimulantes dans les études qui constituent cet ouvrage. Depuis une dizaine d'années, toute une génération de travaux a insisté sur les spécificités urbaines dans la diffusion de pratiques et de savoirs nouveaux. Le thème de la ville, lieu de concentration des élites novatrices, espace privilégié de circulation de l'information et de brassage social, est aujourd'hui de l'ordre du lieu commun. Au-delà de ce constat paré du charme de l'évidence, le rapprochement de la "ville" et de "l'innovation" soulève maints problèmes.

En s'interrogeant sur la diffusion de l'innovation en ville et par les villes - toutes n'étant pas au même titre favorables à son émergence -, l'historien est dans l'obligation de préciser exactement ce qui relève de l'efficace urbain. La constitution de modèles, de familles de villes peut aider au repérage de spécificités, étant entendu que cette élaboration n'a de sens que si elle permet de formuler des hypothèses explicatives des phénomènes d'échanges ou d'interaction, des permanences et des ruptures. Telle est ici la question fondatrice : "Comment écrire une histoire des formations spatiales capable de prendre en compte la régularité des fonctionnements des systèmes et la somme des événements particuliers qui affectent chacun de ses points? » La problématique est rapidement centrée sur l'analyse des processus de diffusion de l'innovation dans l'espace et la société, tour à tour déterminés par les hiérarchies urbaines ou éléments dé structuration de ces dernières. La définition précise de systèmes urbains en Europe, dont tableaux, cartes et graphiques rendent visuellement compte, s'accompagne d'une approche minutieuse de la notion d'innovation trop souvent confondue jusqu'alors avec des termes proches, tels changements ou évolution.

Si les objets d'étude sont ici variés, depuis des technologies " nouvelles" jusqu'aux consommations culturelles en passant par l'implantation d'infrastructures économiques, notons que la nature de l'innovation compte moins pour elle-même que pour la façon dont elle se propage dans le temps et à travers l'espace. Le travail très complémentaire des auteurs associe toujours la mesure de cette diffusion à son interprétation en termes de pratiques sociales, de fonctions et de hiérarchies urbaines. Sans vouloir épuiser la richesse de ce livre, quelques apports nous semblent pouvoir être dégagés. Les contributions aident tout d'abord à mieux définir « l'innovation » comme objet d'histoire. L'appréciation fine de ses modes de propagation constitue une deuxième ouverture; enfin, l'étude des acteurs de l'innovation et de leur champ d'action complète la compréhension dynamique de la diffusion du neuf.

Qu'est-ce donc que l'innovation? Jochen Hoock et Bernard Lepetit adoptent une définition large : " On conviendra ici qu'il s'agit de l'adoption échelonnée dans le temps et dans l'espace, d'un objet, d'une pratique ou d'une idée par des individus ou des groupes liés à des réseaux spécifiques de communication, 
à une structure sociale, à un système donné de valeurs " (p. 8). Celle-ci comporte au moins deux implications. D'une part, l'intégration du neuf dans les usages sociaux apparaît comme responsable de la transformation de la novation en un processus dynamique d'innovation; d'autre part, les processus de diffusion du nouveau peuvent servir à marquer des ruptures et des continuités : l'hétérogénéité temporelle donnée par la longue durée et l'adoption d'une démarche comparative au sein d'espaces suffisamment vastes et représentatifs deviennent des cadres obligés. Les contributions d'Étienne François (p. 59-74) et de Frédéric Barbier (p. 175-196) sur la géographie du livre allemand du $\mathrm{XVI}^{\mathrm{e}}$ au $\mathrm{XIX}^{\mathrm{e}}$ siècle en constituent un bon exemple. Elles soulignent les rapports étroits existant entre les dynamiques démographiques, économiques, voire politiques et le plus ou moins grand développement des activités culturelles. Seule une étude sur le long terme met ainsi en relief les permanences et les mutations d'un espace économique et culturel, autorise les classements typologiques, permet de saisir les décalages entre distribution de la demande et organisation de l'offre.

A ces premières contraintes s'ajoute l'obligation de lever les ambiguïtés de sens et d'interprétation de l'innovation mises en valeur par plusieurs études. La diffusion massive des horloges publiques, symbole du pouvoir urbain et de bon gouvernement à partir de 1370 , illustre le passage de motivations emblématiques au souci de répondre à des exigences économiques ou administratives. Cette émergence d'une " conscience laïque du temps " qui se lit dans les rythmes et les espaces de diffusion des horloges (les villes marchandes et textiles d'Europe) marque une différence entre l'implantation d'une innovation et son intégration dans un champ de valeurs et de pratiques (Gerhard Dohrn Van Rossum, p. 29. 43). Les nuances de vocabulaire jouent à plein pour épuiser le sens de l'innovation, jamais univoque et toujours susceptible de subversions par les pratiques réelles. Ainsi l'implantation des caisses d'épargne dans la France de 1818 à 1848, voulue par des notables locaux soucieux de moraliser le peuple par les vertus de l'économie, mais dont le fonctionnement traduit l'existence d'une épargne bourgeoise aux fonctions différentes (B. Lepetit, p. 146-148). Chaque innovation est susceptible de plusieurs niveaux d'analyse et le processus de sa diffusion est rarement neutre.

La détermination des modalités de propagation et d'insertion d'un phénomène dans une société et un espace revient à définir un processus de diffusion social et spatial. Les contributions du recueil se ramènent fréquemment à un modèle simple, une courbe logistique ou en " $S$ " selon laquelle « l'augmentation à chaque instant du nombre des individus qui adoptent l'innovation est proportionnelle à la fois au nombre de convertis et au nombre de ceux qui sont encore susceptibles de l'être " (p. 17). Les courbes de diffusion des théâtres aux Pays-Bas (p. 120-121), des caisses et livrets de caisses d'épargne en France (p. 137, p. 146) obéissent par exemple à ce modèle et en pointent les limites : le schéma descriptif ne dispense pas l'historien du travail d'explication. L'on peut également dégager les principales variables explicatives des processus de diffusion. Deux modeles existent, l'un par contagion entre des unités adjacentes ou au sein de groupes sociaux homogènes; l'autre, hiérarchique, des grandes villes vers les petites, d'un groupe social dominant vers les autres. La combinaison des modèles reflète l'agencement complexe des fonctions et des hiérarchies urbaines (B. Lepetit, 
p. 153-154; Gilles Feyel, p. 89-111). Plus que des régularités générales, les auteurs mettent en évidence les éléments de structuration des processus de diffusion : réseaux commerciaux, flux interurbains, motivations des acteurs... Les variations dans la diffusion renvoient aussi aux significations de l'adoption de la nouveauté : agit-on par communauté d'intérêt, est-on motivé par l'esprit de concurrence ou d'imitation? L'analyse des mécanismes sociologiques doit pouvoir en rendre compte. Mais au-delà du croisement d'indicateurs socio-économiques ou culturels avec la diffusion spatio-temporelle de l'innovation, on risque l'écartèlement entre des micro-analyses attentives aux hommes, aux pratiques sociales et aux mentalités qui supportent les progrès de l'innovation, et des macro-analyses soucieuses de dégager modèles et principes régulateurs. Pour remédier à ce danger, souligné par Jean-Claude Boyer (p. 206), les auteurs proposent plusieurs fois un inventaire des structures formelles de diffusion de l'innovation étudiée comme socle explicatif (cf. Joseph Wisocki, p. 159-172). La complexité de la notion d'innovation, les combinaisons multiples de sa propagation, imposent en complément de décrire les caractéristiques de ses acteurs et des champs à l'intérieur desquels ils agissent.

L'idée d'une ville toujours innovante trouve ici ses nuances. Ainsi joue l'influence de la taille des cités: en France, en Angleterre, aux Pays-Bas vers 1880 , les capitales, principaux foyers démographiques et économiques, "vitrines» du pays, sont les premières villes équipées par le téléphone (J.-C. Boyer, p. 200). Mais une seconde phase à la fin de la décennie voit les modalités de diffusion entre les villes des trois États diverger pour des raisons fonctionnelles. L'innovation téléphonique, qui joue un rôle de structuration de l'espace économique, souligne les mutations en cours de la hiérarchie urbaine sous l'effet de la révolution industrielle : les cités d'un bon rang démographique mais peu touchées par l'industrialisation sont ici lanternes rouges. Une constatation identique, mais cette fois au profit des villes tertiaires, peut être faite concernant les innovations culturelles en France et en Allemagne du $\mathrm{XVI}^{e}$ au $\mathrm{XIX}^{\mathrm{e}}$ siècle.

Selon qu'on rattache les motifs de l'adoption de l'innovation aux structures urbaines ou aux flux interurbains, les explications, largement déterminées par la problématique de l'auteur, sont endogènes ou exogènes. L'interrogation porte alors sur l'action des États, des institutions, des groupes sociaux qui vont promouvoir ou freiner la diffusion de l'innovation. Il convient de reconstituer les logiques à l'œuvre : ainsi, les rythmes et formes de propagation du téléphone en Lorraine, entre 1885 et 1914 , sont d'abord motivés par les besoins des élites économiques locales avant d'être modelés par un souci moindre de rentabilité mais plus affirmé de service public incarné par les élus et notables administratifs (Jean-Paul Martin, p. 209-222). Logiques complémentaires ou exclusives, le frein absolu à l'innovation n'est jamais évoqué même dans le cas d'institutions sclérosées comme les universités de l'Ancien Régime, sensibles malgré tout aux débats nouveaux, traversées par des gens susceptibles de constituer en dehors des milieux novateurs; telle est "l'innovation par surcroît (Jacques Revel, p. 87).

Le bilan de cet ouvrage est plus riche et plus complexe encore. Il faut retenir l'intérêt de la documentation graphique et statistique, la volonté de rendre compte 
de la complexité et des spécificités des phénomènes urbains de façon théorique et concrète à la fois. Il faut rendre hommage à la rigueur des auteurs et au travail de direction et de synthèse scientifique réalisé par J. Hoock et B. Lepetit qui font de cet ouvrage une auvre collective au meilleur sens du terme. Si l'articulation de la formalisation et de la monographie n'est pas toujours parfaite, la réflexion est du moins lancée et l'ouvrage constitue une invitation sérieuse à jeter un regard neuf sur l'histoire des villes et des circulations culturelles au sens large.

Vincent MILLIOT.

Joël CORNETTE, Un Révolutionnaire ordinaire. Benoît Lacombe, négociant, 1759 1819. Avant-propos d'Emmanuel LE Roy LADURIE. Seyssel, Champ Vallon, 1986. $15,5 \times 24,430 \mathrm{p}$.

Il était une fois un médecin d'Albi qui découvrit chez un antiquaire la correspondance commerciale d'un certain Benoit Lacombe, négociant en vin à Gaillac : 3890 lettres émises à Bordeaux entre 1783 et 1789 et à Gaillac entre 1799 et 1817. Il était une fois un jeune historien qui se fit confier les trois registres, fouilla supplémentairement fonds notariaux et archives révolutionnaires. Cela donna une thèse et désormais un très beau livre.

On regrettera seulement que l'éditeur ait donné à celui-ci un titre quelque peu trompeur sur la marchandise. Non point que Benoit Lacombe n'ait pas été un révolutionnaire ordinaire, un de ces bourgeois qui se fit jacobin très, très prononcé, puis suivit avec la plus parfaite obséquiosité les différents régimes. Mais la phase révolutionnaire de l'homme est précisément celle pour laquelle il n'y a pas ce qui fait le sel de l'entreprise de Joël Cornette, la correspondance. D'autre part, la préoccupation politique est à l'évidence chez Benoît Lacombe de pure conformité. Elle est d'ailleurs absente avant et après la décennie révolutionnaire. Le militantisme de notre notable nous renseigne moins sur lui que sur l'air du temps: en 1793, l'entretien de la notabilité passait par la présidence de la société populaire "des Sans Culottes à picques", comme elle était passé pour le frère aîné de Benoît en 1788 par le consulat de la ville.

Le très grand intérêt, le charme même, du livre est ailleurs. Il est dans la virtuosité avec laquelle Joël Cornette fait sentir les liens affectifs entretenus entre Benoît Lacombe et ses affaires. Car ce qu'il fait détermine ce qu'il est. Nulle schizophrénie chez lui : il est marchand de part en part, et de façon si passionnée, si émouvante que, le livre refermé, je défie quiconque de ne pas se prendre de sympathie pour Benoit Lacombe.

Et de ne pas être triste avec lui après 1789. N'allez pas croire que la Révolution le ruina. Au contraire, il termine sa vie en 1819 avec un patrimoine accru, une notabilité inentamée, des fils qui ont fait leurs études à Sorèze (ils seront plus tard conseillers généraux et députés). Mais voilà, son patrimoine n'est que foncier et immobilier, ses fils risquent fort de devenir bourgeois. Ce qu'abomine Benoit Lacombe. Il s'est voulu négociant, c'est-à-dire travailleur. Mais le négoce n'a 
pas voulu de lui. En 1783, la tribu familiale l'a envoyé à Bordeaux par où transitait jusque-là le vin expédié de Gaillac jusqu'aux Iles. On a pensé que Benoît pourrait apprendre dans le grand port le négoce et s'installer directement. Mais il arrive au moment précis où la conjoncture du commerce colonial atlantique se dégrade (du moins le pense-t-il; les chercheurs d'aujourd'hui sont plus dubitatifs). Et surtout les grandes familles installées ne laissent pas facilement réussir un nouveau venu aux yeux plus gros que le ventre. Les affaires marchent déjà mal quand la mort du frère aîné le rappelle à Gaillac pour gérer les intérêts de la tribu.

Benoît Lacombe va donc se recroqueviller sur l'horizon gaillacois. Certes, il sait conjurer les périls de la nouvelle législation successorale (il a onze frères et sœurs...), achète force meubles et immeubles, sait attendre en ces temps d'inflation pour se faire rembourser ses créances. Pour tout autre que lui, ce serait le bonheur. Mais Benoît a la tête pleine des rêves atlantiques envolés. C'est un romantique du négoce, un Chateaubriand du vignoble gaillacois. Le destin a voulu qu'il devienne un Père Grandet...

François HINCKER.

Jacques LÉONARD, Archives du corps. La santé au XIX siècle. Rennes, OuestFrance/Université, 1986. 15,5 × 23, 341 p., bibliogr. (« De mémoire d'homme : l'histoire ").

Histoire écologique de la santé au XIX $x^{e}$ siècle, Archives du Corps traite des aspects biologiques de l'existence quotidienne des Français et de leur transformation au cours d'une période qui voit s'ébaucher de nombreux traits caractéristiques de la France présente. Cette entreprise de description systématique de " l'ancien régime de santé » et de son lent démantèlement est aussi une invitation à se dégager des "images d'Épinal » symétriques et simplificatrices dont se satisfont aujourd'hui aussi bien les nostalgiques du "bon vieux temps " que les chantres de la marche irrésistible du progrès. En effet, contre les visions intéressées à juger le présent à l'aulne du passé (auxquelles les historiens n'échappent pas toujours), J. Léonard plaide pour la recherche d'une objectivité, fondée sur la confrontation des sources les plus différenciées possibles. "Dans ce livre, on écartera souvent le rideau des gloses et bravant le reproche de la quête naïve d'une objectivité illusoire, on étalera des textes provenant de regards différents, on rassemblera de préférence les faits incontestés, et de ces phénomènes dont on ne peut douter qu'ils ont existé, on osera se dire : " alors, c'était ainsi " " (p. 10).

La démarche ainsi exposée situe l'ambition de l'auteur et circonscrit les limites de l'ouvrage, le constat prévaudra sur l'interprétation. De fait, Archives $d u$ corps offre une source incomparable de renseignements, de données sur l'évolution de certains aspects de la "qualité de la vie" des différentes catégories de la population française. Produit de vingt-cinq ans d'études sur le $\mathrm{XIX}^{\mathfrak{e}}$ siècle et sur l'histoire de la santé, le travail de Léonard est une cuvre de maturité d'une 
grande érudition qui, à travers les inventaires de notaires, les proverbes, les réclames de journaux, les livres «savants", les témoignages littéraires, les rapports des ingénieurs, les enquêtes des économistes, etc., conduit son lecteur, au terme de six chapitres, de l'analyse du bouleversement des rythmes organisant l'écoulement du temps (chap. I) à l'évolution des violences et des souffrances constituant le quotidien du "struggle for life" (chap. vi). Sur ce parcours, il lui donne à voir une population fondamentalement préoccupée de la qualité de l'air qu'elle respire (chap. II), de l'eau dont elle use (chap. III), de son approvisionnement en nourriture et en boisson (chap. IV), de ce qu'elle voit et entend (chap. v). Chaque thème constitue une approche particulière de l'étude des conditions d'existence - comprises ici comme un produit des relations réciproques entre la vie biologique, la vie sociale et l'environnement - et de l'ampleur des transformations qui les affectent, inégale selon que l'on vit à la ville ou à la campagne, que l'on appartient aux élites dominantes, aux couches moyennes, ou aux classes "laborieuses".

En voulant restituer à l'état de santé de la population une place que les approches historiques traditionnelles tendent à négliger dans les analyses qu'elles proposent, J. Léonard ouvre une perspective originale et passionnante. Mais l'esquisse qu'il propose de ce que pourrait être "la préhistoire d'une approche écologique des relations des Français avec leur milieu » (p. 8) suscite deux types de critiques.

D'une part, son livre souffre d'un défaut de construction qui ne tient pas tant au procédé d'exposition thème par thème, dont le souci didactique amène à des redondances obligées (la lecture en est parfois un peu pesante), qu'à l'absence de problématique explicitant le choix et l'assemblage de ces thèmes. L'auteur caractérise son livre comme un dossier incomplet auquel manqueraient de multiples volets, pour s'interdire d'en tirer des " conclusions péremptoires". S'il ne s'agit bien évidemment pas de lui reprocher la non-exhaustivité de son travail, on est en droit de s'interroger sur ce qui fonde la cohérence de l'ensemble qu'il propose. Que ces partielles " archives du corps" puissent, comme si la chose allait de soi, ne traiter ni de la sexualité (sinon pour certaines de ses conséquences particulières, comme les avortements ou les effets néfastes des accouchements multiples) ni de la « folie », suppose une représentation implicite du corps et de la santé concordante avec le découpage opéré par le regard médical qui se construit pendant cette même période. Il est regrettable que le bilan de l'état de santé que J. Léonard dresse en historien ne s'interroge pas sur la logique médicale qui sous-tend son objet et sur les catégories de classement qu'elle induit. Cette remarque méthodologique m'amène à formuler une deuxième critique de portée plus générale.

Il ne suffit pas de braver "le reproche d'une quête naïve d'une objectivité illusoire " pour ne pas l'encourir. Le recoupement de textes provenant de regardsdifférents, par lequel procède dans ce livre l'établissement " des faits incontestés", dispense par trop son auteur d'une analyse des conditions sociales de production des sources qu'il utilise. Faute de prendre en compte les intérêts et de restituer les enjeux qui président aux différentes visions des conditions d'existence des classes populaires, toujours produites de points de vue extérieurs (médecins hygiénistes, folkloristes, écrivains, hommes politiques, etc.), le risque 
est grand de confondre la réalité objective de ces conditions avec ce qui n'en constitue que le sens commun des groupes dominants, c'est-à-dire le misérabilisme, J. Léonard n'y échappe pas toujours.

Patrice PINELL.

Gerassimos Vocos, Philosophia kai ekpaideytike politike (O kartesianismos steu galliké paideia ton $19^{e}$ aiona). Philosophie et politique de l'enseignement. Philosophie et politique dans l'Université française au XIX siècle. Athènes, Lôtos, 1987. $14 \times 22,130 \mathrm{p}$.

La philosophie française universitaire du $\mathrm{XIX}^{\mathfrak{e}}$ siècle est relativement négligée aujourd'hui, et c'est en vain qu'on chercherait chez un libraire un livre de V. Cousin accessible dans une édition qui ait moins d'une centaine d'années d'âge. Il est d'autant plus remarquable qu'un universitaire grec, professeur à l'université de Thessalonique, ait consacré une étude à ce sujet, dans une perspective à la fois d'érudition et d'initiation.

La table des matières de ce petit livre donne une idée de l'envergure du projet auquel il correspond. Introduction (du Descartes des "Lumières" au Descartes de Boutroux) - I. L'éclectisme philosophique (Pour connaître V. Cousin - L'enseignement public en France avant la Monarchie de Juillet - La révolution de Juillet et le rôle éducatif de Cousin - La genèse de l'éclectisme spiritualiste - La théorie cousinienne de l'histoire de la philosophie - Les voyages de Cousin en Allemagne); II. La réaction de l'Église; III. La défense de l'université et de la philosophie (La contre-attaque de Cousin Plan du discours - Principes théoriques - Récurrence historique - Plaidoyer pour l'université - Défense de la philosophie); IV. La méthode interprétative de l'éclectisme spiritualiste et sa contestation (L'éclectisme et la philosophie comme matière d'enseignement - La théorie des quatre types philosophiques - La philosophie comme pédagogie morale - L'interprétation cousinienne de la philosophie de Descartes - L'interprétation de Bouillier - La réaction positiviste : L. Liard).

$\mathrm{La}$ suite de ces rubriques permet de comprendre la démarche adopté : il s'agit d'éclairer un problème théorique - la réinscription du cartésianisme dans la tradition historiographique française, qui confère à celui-ci la valeur d'une origine mythique, et permet en même temps de fonder une méthode et de tracer un programme d'études dans le domaine de l'histoire de la philosophie - par le contexte pratique qui a permis à la fois de le poser et de le résoudre. C'est ainsi que, pour caractériser l'entreprise de Cousin, G. Vocos reprend une formule de Bréhier : "Cousin a été le politique de la philosophie." Or qu'est-ce qu'être un politique en philosophie? C'est reprendre tout le discours de la philosophie en le rapportant à des enjeux qui ne sont pas seulement spéculatifs, c'est-à-dire restituer à l'intervention du philosophe la fonction sociale que, consciemment ou à son insu, il remplit effectivement. On comprend que, de ce point de vue, le statut de l'histoire de la philosophie soit crucial : étudier et enseigner l'histoire de la philosophie, ce n'est pas seulement faire revenir en mémoire le passé 
de tout ce qui a été pensé, et ainsi introduire l'histoire dans la philosophie; mais c'est aussi réinterpréter ce passé à partir d'un présent qui est celui de l'histoire vivante, c'est-à-dire introduire la philosophie dans l'histoire. C'est ainsi précisément que Cousin a exploité la philosophie cartésienne en l'actualisant, en vue de la faire servir à sa propre tentative, celle de fonder la philosophie du sens commun qui donne son contenu au programme de l'enseignement public. En devenant une matière d'enseignement dans cette discipline nouvelle qu'est "l'histoire de la philosophie ", le cartésianisme, au-delà de son système théorique spécifique, s'intègre à l'histoire politique française du XIX ${ }^{e}$ siècle, et intervient, d'une manière qui n'est nullement désintéressée, dans le développement de la nouvelle société issue de la Révolution, où l'organisation de l'instruction publique joue un rôle fondamental.

G. Vocos reconstitue la trame complexe des événements, des débats, des dispositifs institutionnels, qui ont permis de parvenir à ce résultat. En particulier, il montre comment, d'une manière paradoxale, c'est à travers sa double relation d'allégeance et d'ignorance vis-à-vis de la philosophie allemande, représentée principalement par Hegel, d'abord exploité puis rejeté, que Cousin est arrivé à ses fins. A ce propos, on peut se poser la question suivante : ce double jeu s'explique-t-il par la duplicité des arrière-pensées et des intentions? Autrement dit, Cousin avait-il complètement prémédité son entreprise, ou bien a-t-il été entrainé par la logique de la situation dont il n'était lui-mème qu'un élément? On sait que sa "carrière" a connu en 1828 un point de rupture et de rebroussement, au moment où la démarche de l'administrateur s'est substituée à celle du philosophe proprement dit. Dans quelle mesure la première étape de la pensée cousinienne a-t-elle été seulement un point d'appui pour la seconde, au sens à la fois d'un prétexte et d'un instrument contrôlé par un calcul concerté ? Peut-être conviendrait-il d'accorder plus de poids aux textes philosophiques, plus nombreux et moins vides de contenu qu'on ne le croit trop souvent, écrits durant cette période préliminaire, et de s'en servir pour comprendre le mécanisme des interventions publiques, directement politiques, faites ultérieurement par Cousin sous la Monarchie de Juillet, au Conseil de l'université et au Ministère de l'instruction publique, au lieu de les expliquer de manière récurrente comme de purs artefacts, vidés dès le départ de toute valeur spéculative. Car Cousin, et à travers lui toute la philosophie universitaire qu'il a fondée, a été manipulé par la politique dont il a été l'agent, tout autant qu'il l'a lui-même manipulée, au sens d'une construction dirigée de manière complètement consciente.

Pierte Macherey.

Julien Freund, Politique et impolitique. Paris, Sirey, 1987. 15,9 × 24, 426 p. («Philosophie politique »).

Ce récent et dense ouvrage de J. Freund, un des quelques maitres de la philosophie politique en France, rassemble des articles et des conférences publiés ou prononcés dans notre pays ou à l'étranger, ainsi que des éléments de réflexion 
inédits. Composé en trois parties : notions, structures, relations, il présente une remarquable unité d'analyse et de ton qui eût pu ètre compromise par les conditions mèmes de son écriture, évoquées plus haut.

Il n'en est rien, et d'abord à cause de la plume de Freund, mise au service d'une pensée dont la densité n'a d'égale que l'originalité et qui s'accomplit dans ce maitre ouvrage. Définissant la politique comme l'activité essentielle de l'être humain, l'auteur désigne l'impolitique comme «... ce qui contrevient à l'intelligence et à la pertinence dans l'action politique ou qui blesse l'esprit et la vocation de la politique ". Si la politique est la faculté pour l'homme en société d'organiser et de protéger cette même société, contre des menaces externes aussi bien que contre des menaces internes inhérentes à la variété des aspirations humaines, alors l'impolitique est la manière de faire de la politique en négligeant ces impératifs et en allant ipso facto à l'encontre de la finalité « incoercible » de la politique.

L'impolitique est donc la mauvaise politique, celle qui méconnait par exemple ce fait essentiel de l'humanité : la société est politique, donnée naturelle et non création artificielle des hommes, ainsi que l'a montré Aristote. Mais depuis Hobbes, la " philosophie dominante " s'est efforcée de démontrer le contraire en fondant la société sur un contrat, pure convention que l'on peut abroger et non donnée immédiate - au sens de l'allemand unmittelbar - de la structuration sociale de cet animal politique qu'est l'homme.

On comprend mieux dans ces conditions l'amplification contemporaine de l'impolitique sous la pression de l'idéologie révolutionnaire. Impolitique par essence, elle n'est pas au service des hommes dans des sociétés existantes; elle prétend a contrario légiférer dans l'abstrait au nom d'une socièté imaginaire. Plutôt que de protéger les sociétés, elle conduit à des ruptures ou des fractures. Or, selon Freund, la confusion entre politique et morale a provoqué l'intériorisation d'une attente, celle d'une société nouvelle dont l'idéologie révolutionnaire permettrait l'édification. Les capacités de critique, de "résistance ", en ont été durablement et profondément affectées : esprits et comportements sont idéologisés au point qu'ils justifient le despotisme.

En effet, l'histoire de l'humanité, que seul l'utopisme tient pour négligeable à moins que l'idéologie n'opère sa réécriture, enseigne que les régimes mis en place au nom de l'idéologie révolutionnaire ont tous versé dans des systèmes totalitaires ainsi que l'avait noté Raymond Aron: «quelques détentions arbitraires (qu'on a raison de dénoncer) sont inséparables de l'imperfection des hommes et des sociétés. Quelques millions de concentrationnaires révèlent un système " !'

L'Occident n'est qu'imparfaitement conscient de ces conséquences de sa propre idéologisation. Il lui manque une lucidité - toute politique ! - pour comprendre que ces régimes, opposés aux nôtres dans leur essence, qui falsifient l'histoire et calomnient le présent, n'ont d'autre devenir que le despotisme, ennemi du sens de la vérité et de la liberté. C'est pourquoi le danger le plus redoutable réside dans la prétention de l'impolitique à faire une toute autre politique, qui dédaignerait normes et impératifs politiques et dont la conséquence serait la

1. Raymond Aron, Polémiques, Paris, Gallimard, 1955, p. 61. 
détérioration des systèmes de relations de l'ordre politique et social - ceux-là mêmes qui nous garantissent contre le dépérissement de toute politique.

Freund dégage alors ces normes et impératifs politiques, concepts fondamentaux de la politique : pouvoir, puissance, négociation, aliénation; tradition, démocratie, égalité et identité. Il le fait sans ignorer deux cadres essentiels de la politique. Celui des antagonismes sociaux, d'une part : les religions, l'éducation, la morale tentent de les maitriser. Celui des liens entre la politique et l'ensemble des activités humaines, d'autre part, ce qui le conduit à élaborer une philosophie du droit et de l'économie.

Concernant cette dernière, la proximité intellectuelle de Freund avec la philosophie politique et l'économie politique allemandes contemporaines est éclairante : il explicite Sombart qui dégage trois types d'économie en une typologie rationnelle et conceptuelle à la fois. Une économie appréciative (richtende) qui établit des principes universels de l'économie à partir de ceux de la société en une démarche hégélienne. Une économie ordonnatrice (ordnende) qui étudie les phénomènes économiques d'une façon scientifique en dépersonnalisant son objet; une économie compréhensive (verstehende) qui saisit le phénomène économique dans sa globalité : économie, société, civilisations.

A partir de ces analyses et en y mêlant des études in concreto, Freund détermine l'existence de trois types principaux : une économie de réserve, une économie d'épargne, une économie d'investissement dont le développement est inspiré par les travaux de $M$. Weber comme de W. Sombart. Il évoque, enfin, l'émergence d'un dernier type dit de prestations, que l'on pourra assimiler à l'économie de services de cette fin de siècle.

La lecture de l'ouvrage s'achève par la détermination d'une vocation du politique : dans la mesure où la politique est responsable de l'aménagement, passablement conflictuel, de l'ordre social, ce dernier s'oppose à l'impolitique qui entraîne les sociétés dans la détresse et de là, dans la décadence. Témoin l'Europe qui, "... en se recroquevillant sur l'espace de ses frontières depuis une quarantaine d'années, a perdu dans l'aventure toute imagination et tout sens de l'efficacité politique. L'union pour l'union est impolitique, parce qu'elle efface les raisons politiques de l'union, faute de prendre en compte la virtualité de l'ennemi " (p. 422).

Admirable ouvrage qui, dans des temps difficiles, ne renonce pas à poser la question fondamentale - politique -, celle du sens.

Tristan LECOQ.

Servir l'État. Colloque tenu à la Fondation Hugot du Collège de France, les 9 et 10 mai 1985, sous la dir. de Françis Bloch-LAINE, Gilbert ÉtiENNE. Paris, Ed. de l'E.H.E.S.S., 1987. $16 \times 24,280$ p. (" Cahiers de l'homme : ethnologie, géographie, linguistique ", nouv. série, 27).

"Servir l'État " fut en 1985 le sujet d'un colloque organisé à l'initiative de F. Bloch-Lainé et de G. Étienne, et dont les travaux sont publiés dans le présent 
ouvrage. Ce thème appelle une réflexion sur l'éthique du service public et du fonctionnaire, sur le rôle de celui-ci au sein des mécanismes bureaucratiques.

Mais en choisissant une démarche descriptive et comparative, en s'appuyant sur l'histoire pour éclairer le présent, les divers participants de ce colloque ont en même temps dressé un vaste tableau des systèmes administratifs, des causes de leur dynamisme et de leur dégénérescence.

La Chine classique a élaboré un modèle presque idéal de bureaucratie, que nous présente $J$. Gernet dans un remarquable article auquel font souvent écho les autres contributions. A partir du Iv siècle, la Chine crée un système administratif qui assure la pérennité de l'empire; ce système est copié par le Japon médiéval, il inspire l'Europe du XIX siècle. Ce qui frappe, c'est sa modernité, que quelques traits mettent en évidence :

- Une puissante morale du service public: la morale confucéenne, qui condamne penchants personnels et intérêts privés, guide réellement les mandarins chinois. La finalité de leur action administrative, c'est « mettre de l'ordre dans le monde, porter secours au peuple".

- Un mécanisme de recrutement et de promotion égalitaire : dès le vir siècle, le principe du concours et celui du mérite s'imposent; l'empereur ne peut s'abaisser en intervenant dans la nomination des fonctionnaires. Le système de la recommandation, entouré de très fortes garanties, permettait par la suite de découvrir les talents cachés, au sein de l'administration. Le système hiérarchique était souple : chacun pouvait être promu comme rétrogradé.

- L'indépendance à l'égard de l'empereur : du devoir de loyauté naquit une tradition d'indépendance et de remontrances; le service des remontrances, dont le rôle était la critique de l'empereur, était particulièrement prestigieux.

- L'initiative et la responsabilité de chacun : l'empereur se donnait pour règle intangible de ne pas intervenir dans les domaines qui n'étaient pas de son ressort; et l'on attendait de chaque fonctionnaire qu'il fasse preuve d'initiative. Mais tous étaient responsables : l'institution du censorat, totalement indépendante, pouvait critiquer ou imposer son veto à toutes les parties prenantes de l'administration.

Le modèle chinois fut d'abord importé par les Japonais, mais la greffe échoua car les féodalités furent trop fortes : les valeurs d'indépendance, de désintéressement, de service de l'État ne purent s'imposer aux grands seigneurs.

En revanche, l'Europe adopta plus tardivement mais avec davantage de réussite certains aspects du modèle chinois. Ces éléments vinrent se greffer sur des institutions administratives et des traditions bureaucratiques solidement établies, qui sont décrites par B. Meynell pour la Grande-Bretagne et par E. Le Roy Ladurie et $M$. Bruguière pour la France. La Grande-Bretagne adopta au $\mathrm{XIX}^{\mathrm{e}}$ siècle le système du recrutement par examens. C'est l'Indian Civil Service qui en prend l'initiative, et par la suite le Civil Service insulaire. La puissance de l'Europe moderne, sa domination sur le monde, permet à l'héritage chinois de se répandre ainsi dans l'univers.

D'autres systèmes administratifs, tel celui de l'Inde, lentement crée, puis perfectionné par le plus grand souverain moghol, Abkar, avec l'institution des mansabdars, s'approchèrent parfois de celui de la Chine. Pourtant, aucun, dans le passé, ne semble égaler en force la bureaucratie céleste. 
Si les héritages nationaux, les particularités de chaque civilisation demeurent très fortes, une sorte de culture bureaucratique universelle s'est également créée, héritière pour une part de la Chine, pour une part de l'Europe moderne, et portée par celle-ci dans le monde entier. Et l'on observe aujourd'hui un mouvement mondial et presque simultané, de contestation des bureaucraties, à propos duquel s'interrogent les auteurs de l'ouvrage.

Les formes concrètes de dégradation du service de l'État sont communes à nombre de pays :

- corruption liée à la médiocrité et à l'érosion des salaires, dans les pays du Tiers-Monde et dans le monde communiste, mais aussi en Europe occidentale, où des mécanismes illégaux de rémunération demeurent secrets ou tolérés; - l'antagonisme entre généralistes et spécialistes : la prédominance des premiers sur les seconds, parfois leur manque de compétence, furent vigoureusement dénoncés en Grande-Bretagne par le rapport Fulton de 1968; c'est aussi la cause de nombreuses difficultés dans l'Inde de Rajiv Gandhi;

- les liens entre l'administration et le monde politique : $F$. Bloch-Lainé dénonce la réapparition sous la $V^{e}$ République d'une forme de "société de cour", tandis que B. Meynell montre que l'ingérence croissante des hommes politiques britanniques dans la promotion des fonctionnaires est une cause majeure du ternissement de l'image du Civil Service outre-Manche.

Ces formes concrètes de dégradation du service de l'État engendrent des interrogations plus générales sur le sens et la finalité de la bureaucratie, à un moment où son emprise sur la société civile parait trop importante : l'Étatprovidence, héritier de l'État mercantile, détruit-il le dynamisme des sociétés? Conduit-il les nations sur des voies périlleuses? Enfin, sous l'apparence de mécanismes égalitaires, n'agit-il pas à l'encontre de la démocratie?

Si les problèmes sont communs, $M$. Crozier montre dans son analyse de l'État à l'américaine, qu'il compare à l'État français, qu'il faut prendre garde aux solutions importées. La lourdeur de l'État américain provient de la multiplicité des règles de droit élaborées par les juges, d'une telle précision et d'un tel nombre qu'elles deviennent paralysantes, tandis que l'administration française est une construction hiérarchique presque immobile et bénéficiant d'un droit exorbitant. Pour réduire le poids de l'Etat dans les deux pays, les méthodes, les démarches ne peuvent être similaires.

Dans cet ouvrage de grande qualité, il manque pourtant un élément essentiel : la construction européenne. Il n'y a pas dans ce livre de réflexion sur les administrations européennes et leurs fonctionnaires. Or, la création et la croissance d'une fonction publique européenne sont une opportunité de tirer les leçons du passé récent, et appellent une éthique nouvelle.

Il en est ainsi du mode de recrutement des fonctionnaires européens : engagés après un concours mais dans la limite des quotas nationaux, ou détachés par leur gouvernement auprès des organismes européens, ces fonctionnaires sont-ils sélectionnés au mieux? Dans le premier cas, le maintien des quotas nationaux, s'il révèle des inquiétudes quant à l'éventuelle hégémonie d'une nation, ne favorise pas la qualité du recrutement. Dans le second cas, les fonctionnaires ainsi nommés sont-ils réellement indépendants? Peuvent-ils faire prévaloir l'intérêt européen ou sont-ils les émissaires de gouvernements nationalistes? 
Le blocage récent d'une institution comme l'U.E.O. pour des questions d'attribution de postes, donne à réfléchir. Cela révèle la difficulté d'imposer une éthique de la fonction publique européenne, fondée sur la notion d'un comportement indépendant et équitable. Sans doute le problème tient-il pour une large part à l'attitude des gouvernements.

La construction européenne est aussi une opportunité de tirer les leçons du passé quant à la croissance des bureaucraties et aux limites que celles-ci doivent imposer à leurs interventions. De ce point de vue, les administrations européennes doivent montrer un respect des économies nationales et des sociétés civiles d'autant plus grand que les nations demeurent encore assez différenciées.

Guillaume LE LouP.

Bernard LEwIS, Le Retour de l'islam. Trad. de l'anglais Tina JOLAS, Denise Paulme. Paris, Gallimard, 1985. $14 \times 23,432$ p. ("Bibliothèque des histoires ").

Spécialiste de l'histoire de l'Orient musulman, notamment de l'Empire ottoman, Bernard Lewis est l'un des principaux orientalistes contemporains. Juif anglo-saxon, professeur à l'Institut d'études du Proche-Orient de l'université de Princeton, il a déjà signé d'importantes contributions à l'étude de la vie politique islamique en Occident, contributions dans lesquelles on ne peut que constater une claire et profonde érudition.

Rassemblant dix-huit essais publiés entre 1952 et 1983, cet ouvrage tente de comprendre de façon historico-critique l'islam dans ses périodes aussi bien classique que moderne; $B$. Lewis cherche à en déterminer la spécificité et la signification en tant que doctrine et réalité politique concrète. Cette religion qui est «dans son principe une religion du pouvoir » (p. 402), a une conception politique à vocation universelle propre à elle. Depuis sa naissance, l'accroissement de l'impact politique de l'islam s'observe dans le domaine de la vie internationale comme dans celui des affaires intérieures de beaucoup d'États. Endormi au cours des derniers siècles, l'islam se réveille et se réactive aujourd'hui pour la réalisation de sa vision du monde et sa propagation universelle : le panarabisme et le panislamisme, dont les Frères musulmans, le F.L.N., Nasser, l'O.L.P., Khomeini, Kadafi, etc., ne sont que des expressions diverses d'un mème phènomène, le « retour de l'islam » (p. 371-403). B. Lewis constate que cette religion est aujourd'hui "très dominante" à l'intérieur des pays musulmans. Elle se présente aussi d'ores et déjà, comme une puissance politique internationale et une "force très puissante" (p. 401) qui peut être un facteur exerçant une action considérable dans la politique internationale même si elle est « encore dépourvue de direction politique" (p. 401).

Sortant du domaine de l'orientalisme proprement dit, B. Lewis esquisse une 
synthèse des diverses inflexions marquantes de l'Orient musulman, confronté à des crises et à des problèmes qui culminent dans le conflit israélo-palestinien.

Dans les parties consacrées à l'islam classique et à la réflexion théorique, B. Lewis cherche à mettre en évidence le rôle majeur du couple « hérésierévolution ", un concept clé qui, appliqué à la pensée traditionnelle, en ferait découvrir nombre de ressorts cachés. Cette thèse va de pair avec l'idée centrale d'absence de conception purement politique de la révolution et de la liberté dans la tradition islamique. S'il est vrai, pour l'auteur, que «l'apparition de l'islam fut une révolution en soi " (p. 33), la pensée islamique n'a pas su développer une doctrine politique de la révolution. Cela à cause de l'absence d'une séparation nécessaire entre la religion et l'État; ainsi, B. Lewis déclare-t-il péremptoirement : "le pouvoir religieux et le pouvoir politique ne font qu'un " (p. 375). Ce qui signifie que dans l'Orient musulman toute révolution contre le pouvoir politique devient inexorablement une révolution contre le pouvoir religieux, contre la religion. C'est la raison pour laquelle, synonyme de sédition (« Fitna »), la révolution n'est pas permise en Islam. C'est seulement sous l'influence de la pensée occidentale que l'Orient musulman s'est approprié le concept moderne de "révolution " (" thawra ") : "la doctrine occidentale du droit de résister à un mauvais gouvernement est étrangère à la pensée islamique " (p. 54). « L'État islamique est en théorie et dans la conception populaire, une théocratie [...] Le souverain était l'incarnation suprême de la loi sainte » (p. 31).

Il en est de même pour la liberté : "Dans l'usage islamique traditionnel, la liberté était un concept juridique non politique " (p. 99). Certes, dans l'islam, il ne devait y avoir ni église ni prêtre, ni orthodoxie ni hiérarchie, ni royauté, ni aristocratie. Il ne devait y avoir ni caste ni rang susceptible de " troubler l'unité des croyants" (p. 33). En outre: "l'esclave n'était plus un bien mais un être humain, avec des droits moraux et juridiques reconnus; la femme, bien que demeurant soumise à la polygamie et au concubinage, acquérait des droits de propriété qui n'ont pas été égalés en Oocident avant les temps modernes, et même le non-musulman jouissait d'une tolérance et d'une sécurité en contraste marqué avec l'ensemble des non-chrétiens dans la chrétienté médiévale - et parfois moderne " (p. 33). Cependant, toutes ces conceptions n'avaient pas un contenu politique proprement dit mais seulement moral. Il reste à savoir dans quelles conditions la pensée islamique a pu développer ultérieurement un tel contenu politique? Pour B. Lewis, c'est la Révolution française qui a été «le premier grand mouvement d'idées de la chrétienté occidentale à s'être imposé à l'Islam " (p. 67).

L'autre thèse importante de ce livre est contenue dans la narration du conflit israélo-palestinien. Farouche défenseur de l'État d'Israël, de sa légitimité historique et politique, et de sa survie, B. Lewis ne refuse pas pour autant la fondation d'un "foyer" aux Palestiniens, étant donné que la survie de l'État juif ne peut être assurée et pose encore un angoissant problème (p. 168-209). Au demeurant : " Les premiers adversaires d'Israël et les plus importants, sont évidemment les Arabes" (p. 242). Voilà pourquoi, " tôt ou tard, il faut trouver une solution qui fournira un foyer aux réfugiés palestiniens" (p. 209). Pour en arriver là, bien que pessimiste, B. Lewis propose la négociation entre 
Palestiniens et Israéliens; même si à ses yeux les Arabes ne cherchent que la destruction de l'État juif, cela "vaudrait la peine d'essayer" (p. 209). Ainsi, l'obstacle principal à la paix au Proche-Orient est constitué par la charte de l'O.L.P. qui vise à la destruction de l'État d'Israël, et aussi par les enjeux internationaux du conflit : " Malheureusement pour les deux parties, leur conflit n'est pas purement local, un certain nombre de facteurs régionaux et extérieurs viennent le compliquer " (p. 192). Toutefois, " si l'existence d'Israël était admise comme allant de soi, le conflit arabo-israélien pourrait évoluer vers une nouvelle phase dans laquelle le problème ne serait plus l'existence ou la non-existence de l'État $[. .$.$] ce serait un problème politique et diplomatique d'un type plus$ traditionnel, une contestation sur des frontières " (p. 201). N'étant pas chrétiens pour la plupart, « les Arabes ne sont pas antisémites" (p. 242).

Le Proche-Orient « ne partage pas le souci obsessionnel de la race qui touche ses voisins en Europe, en Asie et en Afrique [peut-on exclure l'Amérique du Nord?]. Quelque difficile que puisse être le problème de la Palestine, il n'est pas encore empoisonné par le conflit le plus amer de notre temps et en cela il y a un motif, un léger motif d'espoir" (p. 260).

Pour conclure, il est essentiel de noter que ce résumé très schématique n'entend pas donner une idée complète et fidèle de cet ouvrage : nous n'avons retenu que ce qui se rapporte directement à la pensée politique islamique et au problème palestinien. Il aurait fallu beaucoup de pages pour aborder les autres aspects de cet important essai, et faire ainsi la critique indispensable des nombreuses interprétations ou affirmations qui nous paraissent fondamentalement contestables.

En fait, ce qui est frappant et regrettable, dans ces textes, dans les écrits en général de B. Lewis, c'est leur caractère partial; adoptant une méthode (quasi renanienne !) qui combine engagement idéologique et profonde érudition, cet orientaliste laisse paraître néanmoins une indéniable ambiguïté : sans contester à l'auteur sa liberté de ton et son esprit critique, la pertinence de ses thèses reste questionnée par le caractère sélectif des éléments qui fondent son argumentation, et encore plus souvent, par le tour très péremptoire de celle-ci.

Les travaux de B. Lewis ont toujours suscité de vives polémiques chez les historiens orientaux et les orientalistes.

A. Hussain AzIz.

1880-1980. Un siècle d'électricité dans le monde : actes. Premier colloque international d'histoire de l'électricité organisé par l'Association pour l'histoire de l'électricité en France, Paris, 15-17 avril 1986, éd. Fabienne CARDOT, textes en français et en anglais. Paris, Association pour l'histoire de l'électricité en France, 1987. $18 \times 24,452$ p. (« Histoire de l'électricité ").

L'Électricité et ses consommateurs. Actes du quatrième colloque de l'Association pour l'histoire de l'électricité en France, Paris, 19-21 mai 1987, réunis et 
édités par Fabienne Cardot. Paris, Association pour l'histoire de l'électricité en France/Diff. P.U.F., 1988. 17,5 × 24, 312 p. (" Histoire de l'électricité »).

Deux colloques tenus sous les auspices de l'Association pour l'histoire de l'électricité en France sont venus compléter cette entreprise commencée il y a quelques années, qui vise à retracer dans un cadre pluridisciplinaire l'époque électrique des origines à nos jours. Après avoir abordé les questions méthodologiques d'une telle ambition, ainsi que les grandes caractéristiques d'un siècle d'électricité en France, les perspectives ont été étendues au monde pour cette même période 1880-1980. Enfin, plus récemment, après de telles approches que l'on peut qualifier de balisage, l'intérêt de l'A.H.E.F. s'est arrêté sur les problèmes qui ont été liés aux modifications engendrées par l'évolution de la consommation d'électricité. Mais que ce soit dans le cadre d'un colloque international ou dans celui d'un colloque regroupant essentiellement des Français, il faut reconnaître d'emblée que cet échange de points de vue entre universitaires et ingénieurs, qui aurait pu être source de discordances au regard de l'unité de ton, fut une réussite d'intelligence. Les intervenants sont parvenus, au gré de leurs thèmes propres, à créer une espèce d'harmonie syncrétique qui restitue la nature même de l'électricité, à la fois fruit de la science la plus exacte et réalisation industrielle aux effets les plus pratiques.

Dans ces études de l'émergence et du développement de l'électricité en Europe et en Amérique du Nord des lignes forces se dégagent. Sur le plan technique d'abord, alors que l'électricité n'en était qu'au stade expérimental, on observe un formidable échange d'idées parmi ces pionniers d'une énergie nouvelle. En effet, en dépit des particularités nationales, liées soit à la tradition intellectuelle, telle celle qui oppose le démon français d'une rationalisation mathématique des découvertes à l'esprit pratique et pragmatique des Anglo-Saxons, ou soit encore à l'existence d'un mécénat scientifique comme en Allemagne alors qu'il faisait défaut en Italie, on ne peut que constater que le cas de l'électricité confirme une fois de plus que le développement des sciences et des techniques n'est nullement linéaire. Même un esprit aussi génialement inventif qu'Edison ne crée pas ex nihilo, il s'inspire, corrige, modifie, et enfin adapte sa propre invention aux exigences pratiques. On l'a vu certes avec Edison, véritable héros de ces journées, mais encore lors du second colloque, lorsque cet aspect a été fort bien synthétisé à propos de la lampe à arc d'Elihu Thomson.

Et si, comme on le sait, les recherches fondamentales et expérimentales ont été l'occasion d'échanges et de rencontres entre savants et techniciens, la vulgarisation du produit a donné lieu à des confrontations d'ordre similaire, quel que fût l'endroit. En effet, l'électricité a dû affronter la concurrence d'énergies bien implantées comme le charbon si essentiel à la force vapeur dans l'industrie ou bien surtout comme le gaz si souvent en position de monopole pour l'éclairage public et même domestique, bien que le pétrole ait été là un rival redoutable. Les problèmes liès aux techniques de financement ainsi qu'aux modes de production présentent en cette phase initiale de l'histoire électrique de nombreux points communs, que ce soit au Canada, en Finlande, en Belgique 
ou dans l'un des quelconques autres pays modernes de la fin du XIX $x^{e}$ siècle. Partout aussi, le débat sur les avantages et inconvénients du courant continu et alternatif a eu lieu, et la question du choix entre thermique et hydraulique s'est posée, même si souvent ces deux modes de production se sont côtoyés, voire complétés.

C'est alors qu'interviennent ces facteurs particularisants qui peuvent expliquer le caractère spécifique du mode et de l'état de l'électrification d'une région ou d'un pays. En effet, selon qu'un potentiel hydraulique existait, comme au Canada, en Suède, dans les régions alpines ou pyrénéennes, la houille blanche était prédominante, alors qu'à l'opposé, le thermique avait la préférence dans les régions houillères, et ce d'autant plus qu'une clientèle industrielle préexistait comme dans la Ruhr, dans les bassins miniers de Grande-Bretagne, ou bien encore en Bohême.

La taille du marché potentiel fut aussi un facteur considérable de développement ou de freinage. Précisément, on a souvent expliqué le retard français, italien ou espagnol par l'exiguilté du marché de ces pays. Les structures financières ont eu également une part très appréciable dans le processus d'electrification. La présence d'un marché de capitaux solide et aux ramifications internationales étendues fut un atout pour la formation de grands groupes comme General Electric, Siemens, A.S.E.A. ou même Braun-Boveri.

L'industrie électrique a été, dès ses débuts, un secteur requérant de nombreux capitaux investis à long terme, car la rentabilité ne pouvait intervenir que tardivement, en particulier avec l'hydroélectricité. On observe justement que c'est dans les pays où ces puissantes concentrations financières et industrielles ont été entravées pour de multiples raisons, que la nationalisation des moyens de production et de distribution est venue pallier le manque d'initiative privee pour rationaliser et développer cette branche industrielle. Et si, bien sûr, on pense à l'E.D.F. et à son programme nucléaire, les cas de la Grande-Bretagne et de l'Italie sont tout aussi intéressants.

Une seule ombre à ce tableau, la quasi-absence de communications sur l'histoire de l'électricité en Europe centrale et orientale. Car, si les représentants des pays de l'Est n'ont pu se rendre en France, comme il est si fréquent, ne pouvait-on pas trouver dans le monde libre des historiens ou des spécialistes de l'électricité capables de nous dresser un panorama de la situation électrique de ces pays? N'était-il pas légitime, par exemple, de se demander si le slogan, si cher à Lénine, rendant l'électrification comme consubstantielle à la marche du communisme, avait vu comme son apothéose se réaliser à Tchernobyl?

L'électricité est devenue plus qu'un objet intéressant de recherches théoriques et expérimentales parce que l'on a pu en saisir ses applications pratiques. Mais, si elle n'avait pas rencontré l'intérêt des consommateurs industriels ou domesti. ques, ses potentialités révolutionnaires auraient-elles pu voir le jour? Il était donc avisé, après avoir envisagé les aspects généraux du phénomène électrique, d'aborder celui-ci sous un angle plus particulier: l'utilisateur d'électricité. Derrière ce simple mot se dissimule toute une histoire riche et foisonnante. Elle permet de saisir des aspects importants de l'impact de l'électricité sur le développement industriel et social qu'elle a accompagné ou mème suscité.

Si généralement on réalise plus ou moins bien les effets bouleversants de 
l'usage toujours plus maîtrisé de l'électricité dans le domaine des moyens de communication qui ont vu la perception de l'espace et du temps se métamorphoser radicalement avec le télégraphe électrique, puis le téléphone, la radio, etc., on est peut-être moins conscient de l'indispensable rôle que l'électricité a joué rapidement dans l'industrie. Évoquons, entre autres, l'exemple si inévitable de l'aluminium qu'il en devient un véritable truisme; ou rappelons encore la part majeure jouée par l'électricité dans la chimie. A ce propos, une communication fort savante et intelligente a montré à quel point l'électricité était devenue indispensable. L'issue de la Première Guerre mondiale n'aurait-elle pas été différente à partir de 1917 si l'équipement hydroélectrique français avait èté moins développé ? Car l'arrêt des importations de nitrate chilien, nécessaire aux explosifs, engendra un grave problème de ravitaillement, qui fut pallié de justesse par la production d'explosifs de synthèse, à un moment où la reprise de la guerre de mouvements accrut considérablement la demande en la matière.

Pour le particulier, l'électricité ne fut pas non plus sans importance. Elle perdit, sous l'offensive commerciale des sociétés de distribution, ce caractère d'ènergie de luxe, pour devenir toujours un peu plus partie intégrante de la vie de tout un chacun. Et, si elle n'a évidemment pas fait surgir cette vie de rève si vantée par les thuriféraires du "tout-électrique", elle n'en a pas moins été un agent actif de cette révolution des mœurs qui s'est déroulée tout au long du $\mathrm{Xx}^{\mathrm{e}}$ siècle. Bien sûr, cette implantation électrique ne s'est pas faite sans hésitation ou même sans ratés - on songe, entre autres, à l'utopie du labourage électrique. Il a fallu susciter une demande parfois réticente, pour surmonter des "goulots d'étranglement", afin de poursuivre une politique d'innovation vitale dans un contexte de concurrence âpre.

Ce sont donc là les grandes lignes qui se dégagent de ces deux colloques successifs, consacrés à l'histoire de l'électricité. Un phénomène scientifique, technique et social qui présente des similarités dans son mode d'apparition et d'implantation, par-delà les disparités géographiques, économiques et politiques. Si des particularités existent en fonction des données précédentes, elles ne doivent pas dissimuler - pour intéressantes qu'elles soient en elles-mêmes - cet aspect uniformisateur de l'électricité. En bien des points, il faut tenir ces conquêtes de la technique comme un des grands acquis positifs de ce siècle, même si les effets annexes n'ont pas toujours revêtu les couleurs séduisantes qu'annonçaient les prophètes infaillibles de la fée Électricité.

Philippe BONOLAS.

Marianne AMAR, Nés pour courir. Sport, pouvoirs et rébellions (1944-1958). Grenoble, Presses universitaires de Grenoble, 1987. $16 \times 24,232$ p., annexes ( Sport en questions »).

En publiant la thèse de doctorat qu'elle a préparée et soutenue dans le cadre du Centre d'histoire de l'Europe du vingtième siècle de la Fondation nationale 
des sciences politiques, Marianne Amar nous livre le résultat d'une enquête sérieuse, fondée sur une solide étude des archives disponibles, dont les documents publiés en annexe offrent un bon échantillon. Le fait mérite d'être précisé, car l'auteur n'a pas échappé à la tentation des effets de style, au plaisir de l'écriture, au goût de la formule à l'emporte-pièce, qui rendent certes attrayante la lecture de l'ouvrage, mais apparaissent souvent comme une virtuosité gratuite, l'ajout d'une verroterie quelque peu clinquante à un texte qui n'en avait guère besoin et qui se trouve ainsi plus desservi que mis en valeur, si l'on ne prend pas la précaution de constater que, derrière le brillant de l'expression, se dissimule de fait une recherche solidement étayee.

Cette remarque faite, la thèse de Marianne. Amar mérite d'être connue et discutée. Pour elle, en ce domaine comme en beaucoup d'autres, la IV République a échoué, et il était quasi impossible qu'il en fût autrement, tant le sport apparaît étroitement lié à la culture de masse de la société française de l'époque. Symbole voulu et recherché d'une République nouvelle désireuse de sortir des ornières du passé, le sport fait l'objet d'un discours convaincant, comme moyen éducatif privilégié qui permettra au régime de créer cet homme nouveau, âme saine dans un corps sain. Mais le discours n'est guère suivi d'actes. De la reconstruction économique aux guerres coloniales, le régime a bien d'autres priorités et l'équipement sportif, fort insuffisant au sortir de la Seconde Guerre mondiale, ne connaîtra guère jusqu'en 1958 cette expansion promise au lendemain de la Libération. Même échec en ce qui concerne le renouvellement des hommes; après les brefs effets d'une épuration sans rigueur, l'organisation sportive retrouve ses dirigeants traditionnels et le sport son rôle de spectacle, l'opinion publique se passionnant pour le champion-vedette beaucoup plus que pour la participation active. Marianne Amar montre avec brio comment, pour un peuple qui a connu l'amertume de la défaite, les rigueurs de la pénurie et qui fait l'apprentissage des seconds rôles après avoir longtemps joué un rôle déterminant dans le sort du monde, le champion remplit une fonction de compensation : champion du monde de boxe, Marcel Cerdan venge le pays des coups reçus durant l'Occupation et Louison Bobet, le héros souffrant, mais malgre tout vainqueur, fait figure d'archétype du destin national.

Ayant échoué dans sa mission éducative, le sport n'échappe guère à la détermination du politique, ni dans les pratiques de ses dirigeants qui reproduisent jusqu’à la caricature manœuvres électorales et tactique du noyautage, ni dans les divisions qui l'affectent, ni dans sa dimension internationale. La guerre froide aura là aussi des répercussions, les communistes faisant connaître leur refus du sport capitaliste par leur mainmise sur la F.S.G.T. (dont Marianne Amar a pu consulter les archives et qui nous vaut un des meilleurs chapitres du livre sur les "mollets rouges"), cependant que les socialistes créent une bien faible Union sportive travailliste. Enfin, loin de contribuer à la bonne entente entre les peuples qui figure dans le catalogue des bonnes intentions des dirigeants sportifs, il sert d'exutoire et de moyen à travers les rencontres internationales à l'affrontement entre nations, la compétition ainsi ouverte réservant à la France plus de sujets d'amertume que de motifs de satisfaction. C'est donc au total l'histoire d'un échec que décrit Marianne Amar. A l'issue de la lecture de ce 
livre riche, intelligent, fécond en perspectives neuves, on est cependant conduit à s'interroger. Les pratiques de la IV' République (mais faut-il lui en réserver l'exclusivité ?) ont sans doute échoué, mais quelle était la norme, la recette magique qui aurait permis qu'il en fût autrement? Peut-on concevoir qu'une pratique sociale réponde à des lignes de force différentes de celles qui meuvent la société dans laquelle elle prend place? On rétorquera (avec quelque raison) que ceci est un tout autre problème qui dépasse peut-être les compétences de l'historien.

Serge Berstein.

Gèraldi Leroy, Anne Roche, Les Écrivains et le Front populaire. Paris, Presses de la Fondation nationale des sciences politiques, 1986. 13,4 × 22, $326 \mathrm{p}$.

Il était tentant en ce cinquantième anniversaire du Front populaire de s'interroger sur la manière dont les intellectuels (l'échantillon est ici réduit aux écrivains) avaient ressenti l'événement. C'est l'entreprise qu'ont tentée Géraldi Leroy et Anne Roche en nous proposant une série de monographies évoquant les divers aspects de l'engagement des écrivains dans le Front populaire, puis l'impact de celui-ci sur leurs ceuvres purement littéraires. Notons d'emblée, pour le regretter, qu'à aucun moment l'ouvrage ne se propose de répondre à la question fondamentale qu'il suggère : pourquoi, au tournant des années trente, tant de romanciers, d'essayistes, de mémorialistes ont-ils éprouvé le besoin de s'engager en politique, de s'instituer les maitres à penser de leurs contemporains, de leur indiquer la voie à suivre dans la vie publique? Quelle conjoncture assez pressante pousse ainsi l'écrivain à sortir de son champ d'action pour s'aventurer sur le terrain piégé de la politique?

Question sans réponse dans l'ouvrage, et qui, du même coup, en diminue singulièrement l'intérêt. Il reste un livre en deux parties fort différentes par leur structure. Dans la première partie de l'ouvrage, nos écrivains se font combattants des armées déjà constituées au sein desquelles ils s'engagent. Nous apprenons ainsi sans surprise qu'autour de Maurras et de Daudet les écrivains traditionalistes ressassent interminablement dans L'Action française les thèmes obsessionnels de l'extrème droite, que parmi les jeunes la tentation du fascisme s'exerce dans des revues relativement proches les unes des autres comme Je suis partout, Combat ou l'Insurgé et que Drieu la Rochelle voit dans le fascisme la panacee universelle. Dans le camp opposé, Marianne ou Vendredi rassemblent les bataillons d'écrivains chez qui l'antifascisme l'emporte sur l'anticommunisme, cependant qu'à Esprit ou Europe se regroupent ceux qui souhaitent prendre position sans pour autant accepter la discipline d'une formation politique organisée. Enfin, la pléiade des intellectuels révolutionnaires opposés au stalinisme ou des écrivains prolétariens non engagés dans un parti critiquent la timidité du Front populaire et s'efforcent de l'entrainer sur le terrain des grandes réformes de structure. Disons-le clairement. A quelques nuances près (les écrivains prolétariens), cette première partie du livre ne nous apprend rien qui 
ne soit depuis longtemps connu sur la participation des écrivains au combat politique. Il aurait été intéressant de savoir si l'engagement des intellectuels débouchait sur des pratiques d'action spécifiques, si une réflexion plus nuancée résultait de leur intervention, s'ils coloraient différemment par leur seule présence la nature des luttes de partis. Le problème n'est guère abordè. Mais, au fil des citations, on ne voit guère l'apport original des écrivains à la famille de pensée qu'ils ont décidé de soutenir. Le Drieu de l'Émancipation nationale n'est qu'un journaliste sans qualité particulière, qui répète ce que son parti attend qu'il dise, et Vendredi meurt d'avoir voulu ignorer les contradictions intellectuelles d'une coalition de partis qui ne pouvait survivre qu'à condition de faire l'économie des analyses qui sous-tendaient son action.

La seconde partie du livre est incontestablement plus neuve puisque les auteurs se proposent de rechercher la marque du Front populaire dans les œuvres de fiction écrites depuis 1936. La première remarque à faire dans ce domaine est que cette période de l'histoire a inspiré un nombre relativement restreint d'œuvres littéraires, et la remarque vaut aussi bien pour la période qui suit immédiatement l'événement (1936-1949 pour les auteurs) que pour les "représentations tardives " des années 1950-1984. Et l'on est frappé de constater que, dans les auvres les plus anciennes comme dans les livres les plus récents, ce sont les mythes véhiculés par le Front populaire beaucoup plus que l'histoire de celui-ci qui sont proposés au lecteur. Au fil des romans analysés dans le livre défile une étonnante galerie de stéréotypes, sans que les travaux historiques conduits sur cette période relativement bien étudiée et qui pourraient amener à nuancer le manichéisme des images figées dans leur immuable fixité paraissent jouer le moindre rôle. Goût des stéréotypes qui vaut pour la vision de droite comme pour l'interprétation de gauche du Front populaire, chacun disposant des siens, à telle enseigne que la simple description du gréviste, du patron ou du militant permet immédiatement de savoir de quelle lecture politique de 1936 se réclame l'auteur. Euvres peu nombreuses, vision conformiste des choses, les auteurs ont sans doute raison d'affirmer que le Front populaire n'a fait naitre aucune grande œuvre littéraire.

Et on ne peut que s'étonner avec eux qu'une période qui a vu les écrivains s'engager si massivement dans le combat politique, se faire éditorialistes, orateurs de meetings, collecteurs de pétitions, témoins ostensibles des camps opposés dans un combat sans merci, ait laissé si peu de trace dans l'activité professionnelle et dans l'œuvre de ces intellectuels. C'est sans doute que les phases et les rythmes de l'histoire culturelle, relevant d'un domaine qui est celui des valeurs, des modes de sentir et de penser, de la sensibilité et des structures mentales, obëissent à des déterminations qui ne sauraient épouser étroitement les séquences de l'histoire politique. Et peut-être à ce niveau, le Front populaire a-t-il été vécu par ces écrivains comme un moment qui les engageait profondément en tant que citoyens, mais non comme une rupture culturelle assez profonde pour qu'elle entraine de leur part la recherche de formes nouvelles ou que leurs livres soient pénétrés des heures fiévreuses qu'ils vivaient dans les réunions populaires ou les salles de rédaction des revues hebdomadaires. Peut-être?... Car sur ce point, sauf quelques phrases fugitives dans la conclusion, le livre est muet. C'est dire que l'ouvrage de Géraldi Leroy et Anne Roche, s'il apparait comme une tentative 
utile de défrichement d'un terrain peu exploré (au plan des cuvres de fiction) ne constitue pas cette histoire culturelle du Front populaire qui reste à faire.

Serge Berstein.

Francis AfFERGAN, Exotisme et altérité. Essai sur les fondements d'une critique de l'anthropologie. Paris, P.U.F., 1987. 13,5 × 21,5, 295 p. ("Sociologie d'aujourd'hui ").

Dans ses visées épistémo-critiques, l'ouvrage remet en cause directement les a priori constitutifs du métier d'ethnologue. C'est une sanction mais aussi bien un cri "pratique" au sens kantien, qui déchire le silence des panthéons positivistes et bouscule les hagiographies des pères fondateurs consacrés par la profession. Francis Affergan questionne les pratiques effectives d'hier et d'aujourd'hui. Il dénonce la réalité empirique du terrain, louvoyant entre deux écueils : d'une part, l'aliénation de l'observé, objectivé et "chosifiè " dans la forme d'un modèle descriptif préetabli et d'un questionnaire de type policier; d'autre part, l'aliénation de l'observateur scientifique dont l'identité se dissout dans l" " observation participante ». Mais la rationalisation théorique qui ordonne le traitement et la classification des données d'observation est plus encore contestée aux niveaux de ses requisits et de ses effets : l'irréductible singularité de l'Autre est sacrifiée au profit des formes multiples de l'appropriation "identitaire". L'indigène ou la culture exotique est soumis à un étalonnage savant qui le réduit au "Même", en lui assignant une place dans une logique de la « différence » et donc de l'assimilation. La différence, schème conceptuel qui identifie l'indigène à une somme d'attributs qui varient du plus au moins, s'oppose terme à terme à l'altérité. Celle-ci serait la radicale étrangeté, celle qui déjoue tous les pièges annexionnistes ou impérialistes et tous les comparatismes dévalorisants.

Pour argumenter sa critique des fondements de l'anthropologie, l'auteur propose une généalogie du regard ethnographique dont la théorie de l'altérité et ses multiples " perversions " constituent le sol originaire et « transcendantal ». Le texte intéresse à ce titre l'historien et le philosophe des sciences qui ne manqueront pas d'en commenter la périodisation et les présupposés. Ce n'est pas amoindrir les qualités d'une problématique extrêmement rigoureuse et documentée qu'en marquer les limites, réciproques de son efficacité propre: l'usage critique des sources historiques a pour fonction d'exhiber des " fondements " conformes aux thèses qu'on veut produire. C'est un procédé éprouvé, il a ses règles, Francis Affergan n'y déroge pas.

Au commencement donc était l'altérité, née du choc de la rencontre des voyageurs occidentaux avec la réalité exotique du Nouveau Monde. Le Xvir siècle témoigne de ce "désir de l'Autre", de cette curiosité pour la "merveilleuse variation" de l'homme dont les mobiles se résument mal dans une volonté d'asservir, de dominer ou d'exploiter les nouveaux espaces ouverts à l'investigation. Ce «désir de l'autre" autorisait et recommandait une conscience exotique, 
respectueuse des particularismes culturels. Mais l'impossibilité de dire, de restituer par écrit l'événement de li rencontre, va ruiner dès l'origine la possibilité d'un dialogue constructif. Faute de réciprocité, le sujet de l'énonciation ethnographique ou le narrateur des relations de voyages va "objectiver» le sauvage en le comparant, sur un mode autoréférentiel, au parangon de toute humanité vraie, l'homme blanc, occidental, chrétien. Le $x^{e} I^{e}$ siècle, moment de la découverte, apparaît alors rétrospectivement comme le siècle des rendez-vous manqués. Le voyage aux Amériques avait pour finalité de renouer le dialogue avec les origines, de reconstituer une totalité de sens où l'autre complète, par son écart intrinsèque, l'unité d'un ordre divin. Le voyage abolit le désordre du temps en exaltant le caractère complémentaire des mœurs et coutumes. Toutefois, il apparaît vite que l'altérité est l'autre nom de l'indicible et du chiffre secret : déficit des mots devenus inutiles ou insignifiants, carrousel des sensations, catastrophe de la subjectivité qui perd ses repères. La vision de l'Autre ne trouve pas ses mots. Le narrateur invite son lecteur à le croire parce qu'il a vu.

Rabattue sur les modèles éprouvés d'une rationalité instrumentale et d'une logique classificatoire, l'altérité va subir une étrange phagocytose pour signifier, au rebours de toute reconnaissance, sa "différence ". En fonction de critères aussi discriminatoires que restrictifs, la valeur du sauvage se découvre rapidement tributaire de l'efficacité utilitaire qu'on lui prête : l'indigène se soumet facilement, sa force de travail est exaltée, on peut l'évangéliser, etc. Le xvII ${ }^{\mathrm{e}}$ siècle donnera à cette rationalisation l'appoint de ses concepts "scientifiques". L'unité de l'espèce, très significativement mise en avant par Buffon, renforce cette « pseudoidentité hétéro-conférée" en légitimant tous les processus d'acculturation et d'assimilation. Dès que l'altérité cesse de valoir pour un ordre unique en son genre, respectable pour soi, le comparatisme peut fragmenter l'objet et lui imposer un classement qui le soumet au jugement objectif, savant : les sauvages selon les cas pourront être soit valorisés («ils sont comme nous ") soit dévalorisés ( «e sont des bêtes »). Mais valorisation et dévalorisation participent du même mouvement qui intègre des variables segmentées dans une échelle normative. La pseudo-objectivité des jugements impose encore que l'énonciation à dimension subjective, dominante dans les anciens récits de voyage qui affirment en première personne ( $\mathrm{J}$ 'ai vu»), s'efface lentement derrière un énoncé plus technique, plus globalisant et toujours plus référentiel (les indigènes sont ceci, font cela...). A partir du moment où l'anthropologie ne se contente plus de présenter une culture étrangère mais la re-présente en s'efforçant de la comprendre et donc de la penser, l'arbitraire de la subjectivité se fait d'autant plus insidieux. Non seulement le sujet disparait subrepticement de la narration mais le discours strictement descriptif et dénotatif se confond avec ses modalités interprétatives et normatives (ex. de la couleur). Il en découle plusieurs conséquences qui sont autant d'apories de l'anthropologie actuelle selon F. Affergan :

1. Le statut de l'observateur n'est pas questionné. Comme dans l'empirisme le plus classique, on le considère comme un simple miroir réfléchissant du monde social appréhendé. Sa neutralité affichée est la condition de l'objectivité de ses informations. Or cette soumission béate au réel est une chimère épistémologique.

2. Le vœu de tout voir est une illusion. Le sujet qui observe sélectionne les 
traits pertinents en fonction de ses a priori. Plus encore, il reste tributaire de ses affects comme de ceux de son vis-à-vis exotique.

3. Le sujet qui pretendument s'efface pour laisser parler les choses construit en fait l'être de ce qu'il voit en fonction de son vécu empirique, en fonction des représentations et du langage conceptuel qu'il véhicule, en fonction des situations symboliques qu'il intériorise.

4. Pour échapper à l'accusation de malfaçon ethnocentriste, l'anthropologue postule l'identité de nature entre les opérations mentales du sujet de la connaissance et de son objet; par-là est affirmée l'universelle adéquation consciente ou inconsciente - des activités classificatoires de l'indigène et des ethnologues. Il suffirait alors de comprendre le mécanisme de ces classifications pour accéder au sens intrinsèque, à l'être des peuples étudiès. Pour F. Affergan cette "compulsion à classer" est une forme symptomale de " transfert narcissique ».

Ces présupposés laissent percer les fonctions prescriptives du texte ethnologique classique dans ses dimensions normatives, pédagogiques ou judicatoires. L'autre est ravalé au rang d'accusé : il doit avouer, on peut avoir prise sur lui ; son statut est déjà fixé. C'est « un même en puissance asymptotique ». La mauvaise conscience de l'ethnologue, née de son rapport ambivalent à la culture occidentale, est à la mesure de sa bonne conscience. Il faut critiquer, selon F. Affergan, l'jmage du couple pervers (civilisé $v s$ sauvage), fruit de toutes les lectures binaires, qui ferait de l'Autre une victime ou un semblable naturel et originaire.

Comment sortir de la crise des savoirs ethnologiques? Pour l'auteur, «l'Autre est et véhicule la possibilité d'une expérience antéprédicative ». Tant qu'on plaquera sur sa réalité des catégories toutes faites, le piège des attributions sommaires et des ratiocinations distanciées se refermera sur l'ethnologue le mieux intentionné. L'ethnologie a trop longtemps monologué, il lui faut accepter les conditions d'un dialogue constructif où le sujet observateur accepte de se mettre à distance de lui-même, pour mieux accéder à l'irréductible altérité de son interlocuteur. Celui-ci deviendrait co-auteur d'un texte polymorphe dont la langue serait plus et moins qu'un code structural : une authentique relation, dialoguée et risquée, offerte au pluriel des lectures. Les valeurs auto-conférées s'effaceraient devant la prégnance du fait qualitatif, des paroles, des visages, des regards, etc.; les "signifiances" du donné empirique surgiraient de la collision des univers linguistiques portant avec soi la nécessité de locuteurs en situation de réciproques échanges. Ceci impliquerait très évidemment, d'une part que l'Autre ne soit pas manipulé par le jeu des questionnaires standardisés ou inadéquats, d'autre part que le professionnel du terrain ne soit pas "signifiè " lui-même dans sa posture d'enquêteur. Revendiquant le principe dialogique de $M$. Bakhtine et l'enseignement des phénoménologues, F. Affergan affirme que le «Je" de l'ethnologue s'appréhende face à l'Autre en le posant. Le « Nous" dialogique réduirait à rien la possibilité d'une déduction rationnelle des catégories anthropologiques. L'événementiel devrait précisément être saisi au niveau de l'expérience de l'espace propre à l'indigène et dans la duree spécifique de sa langue. A ce titre, l'Autre sera une personne et non un simple individu passible d'une 
expertise plus ethologique qu'ethnologique. L'un et l'autre seront ainsi reliés, ce qui n'est pas dire confondus.

Il appartient bien sûr à la communauté scientifique visée de répondre à cette interpellation critique. Le style philosophique très technique de l'auteur fera sans doute barrage à sa réception large. Les questions qu'il pose sont pourtant essentielles. Les dimensions politiques, utilitaires, de l'ethnologie scientifique qu'il met à plat sont souvent considérées comme des épiphénomènes douloureux mais inévitables du travail scientifique. Or F. Affergan juge qu'elles représentent des corrélats presque obligés de l'idéologie rationaliste des ethnologues. Le débat est donc urgent et grave. A un autre niveau, l'ouvrage est une source de réflexion pour les philosophes qui trouveront ici un dossier "en situation" sur un thème mobilisateur qui avait reçu de La Logique du sens de G. Deleuze une approche dècisive.

L'historien des sciences anthropologiques est sans doute plus réticent. La périodisation est dans ce texte soumise à l'évaluation d'une pensée critique qui s'autorise des récurrences discutables. C'est là sa limite réelle. L'histoire est ici simple instrument d'une thèse. Le saut et les retours non justifiés des voyageurs $\mathrm{du} X V \mathrm{I}^{\mathrm{e}}$ à ceux $\mathrm{du} X V I I^{\mathrm{e}}$ siècle puis aux théoriciens presque contemporains ne s'expliquent que par les attendus critiques de l'auteur. Celui-ci propose une périodisation axée sur la réduction de l'altérité par une anthropologie des différences qui devra, dans l'état inchoatif des études historiques, être discutée. D'une part et, après tout, rien n'indique absolument que l'altérité soit l'objet unique de l'anthropologie, comprise dans la longue durée, même si c'est un butoir épistémologique pour notre époque. D'autre part, à réduire ce concept à son acception phénoménologique, F. Affergan fait de l'expérience de l'altérité, une fracture psycho-existentielle toujours première, à la fois déréalisante et constitutive. Pour cette raison, et parce qu'elle ne saurait être cumulative, c'est une expérience précisément sans histoire. Mais peut-on «croire» que, de Christophe Colomb à Victor Segalen, la rencontre avec l'indigène se réduise à ce va-et-vient d'un regard vierge de toutes références, à ce désir originel de l'Autre, qui s'accomplit sans même pouvoir énoncer autre chose que son trouble affectif? Et comment, si tel est le cas, convertir ce regard exotique en concept opératoire? L'auteur veut prouver que le $x^{\mathfrak{e}}{ }^{\mathfrak{c}}$ siècle n'en pré-jugea pas le savoir, qu'il demeura de ce fait réceptif et ouvert à la culture indienne. Il est vrai que quelques citations choisies attestent cette disponibilité des regards et des esprits. Mais dans d'autres cas, l'expression des idées théoriques parait forcée, superlative, par comparaison avec les exemples proposés. Autant dire que certains arguments jugés " fulgurants ", révélateurs d'un impensé, chargés d'effets de sens, n'emportent qu'incidemment la conviction du lecteur bienveillant. Malgré cette restriction qui mériterait plus ample commentaire, l'ouvrage de Francis Affergan associe la réflexion à la rigueur documentaire. Ses qualités formelles le recommandent encore à tous les lecteurs exigeants qui se satisfont mal des rhétoriques à courte vue. C'est un travail de pensée. A son tour, il fait penser.

Claude Blanckaert. 
Elisabeth ROUDINESCO, Histoire de la psychanalyse en France. La bataille de cent ans. T. I : 1885-1939, nouv. éd. revue et corr.; t. II : 1925-1985. Paris, Seuil, 1986. $16 \times 24,498$ p., index, bibliogr. et 783 p., pl.

Au premier abord, ces deux volumes d'Elisabeth Roudinesco s'apparentent à une saga romanesque ou à un film en technicolor : ils multiplient les anecdotes personnelles, de façon à faire de l'histoire de la psychanalyse en France une « histoire » précisément, ou plutōt des histoires, avec leurs personnages et le drame d'existences singulières représentées dans leur vérité concrète; en effet, selon le propos mème de l'auteur, il s'agissait d' « imaginer la vérité sans céder à la fausse transparence d'un énoncé d'archives " (t. II, p. 116), en produisant " une espèce de roman à mi-chemin de la restitution intégrale des faits et de la nécessaire invention de l'histoire " (ibid.). C'est cet aspect, à la fois factuel et fabuleux du matériau brassé dans cette somme qui a d'abord retenu l'attention des lecteurs, et nourri les polémiques que, par sa nature même, une telle entreprise devait déclencher. Toutefois, il ne faut pas s'y tromper : à les lire de plus près, les deux livres d'E. Roudinesco ouvrent un domaine d'investigation qui, à travers l'histoire des idées au sens large, concerne aussi l'histoire de la pensée philosophique et scientifique; et de ce point de vue leur véritable place se situe à côté de ces classiques comme, par exemple, Spinoza et la pensée française avant la Révolution de P. Vernière (P.U.F., 1954, rééd. 1982) et l'Introduction du darwinisme en France d'Y. Conry (Vrin, 1974). En effet, à la suite de ces deux ouvrages, E. Roudinesco reprend la question de "l'introduction " d'une forme de pensée théorique dans un autre contexte que celui dans lequel elle s'est d'abord enracinée.

Deux choses caractérisent une telle introduction : d'abord, il s'agit non d'un acte élémentaire et instantané, mais d'un processus complexe, qui s'étend dans le cas considéré sur près de cent années, et fait intervenir des déterminations qui se tiennent à des niveaux très différents. D'autre part, la "connaissance " doctrinale que développe ce processus met en jeu complémentairement et concurremment un phénomène de reconnaissance et un phénomène de méconnaissance, l'un n'allant pas sans l'autre. De ce dernier point de vue, la thèse défendue par E. Roudinesco est radicale : "Il n'existe pas de "bonne" assimilation d'une doctrine, mais seulement de "fausses " reconnaissances, faites de visions déformées " (t. II, p. 20). "Introduire" une pensée, en lui imposant les conditions de sa " naturalisation ", c'est la transformer : l'histoire de la pensée hégélienne en France, à laquelle E. Roudinesco fait plusieurs fois référence, est exemplaire à cet égard. Mais la compréhension de ce phénomène redouble l'intérêt de l'analyse qu'on en fait : car les obstacles et les résistances que suscite l'effort pour introduire une pensée étrangère servent du même coup d'irremplaçables révélateurs en ce qui concerne les tendances profondes qui travaillent le terrain d'accueil sur lequel on cherche à la transplanter. Les livres d'E. Roudinesco présentent, en fait, au miroir de la psychanalyse, une histoire complète de la pensée française au cours de ces cent dernières années; et sur cette histoire, si nous disposions d'études partielles, faisait encore dèfaut une perspective 
d'ensemble, dont l'histoire de la psychanalyse, si surprenant que cela puisse paraître, permet de tracer l'allure générale.

L'histoire que reconstitue E. Roudinesco n'est donc pas neutre, comme l'est trop souvent une simple histoire des idées, analysée en termes de "pénétration » et de "diffusion"; mais elle fait ressortir des enjeux. Quels sont les enjeux de la reconnaissance de la psychanalyse en France? Ils sont de quatre ordres: therapeutique (en rapport avec un "savoir" de type médical), théorique (en rapport avec des présupposés philosophiques), poétique (en rapport avec certaines pratiques de la littérature), politique (en rapport avec une conjoncture générale, mais aussi avec des formes organisationnelles, puisque la psychanalyse est aussi une institution, et a été gérée comme telle, en France et ailleurs). L'intérêt des analyses présentées dans cette histoire de la psychanalyse en France est de montrer comment ces différents types d'enjeux interfèrent, d'une manière qui n'est pas toujours concordante. Nous disions tout à l'heure que l'introduction d'une pensée théorique dans un milieu étranger est un processus complexe : nous pouvons à présent aller plus loin, en postulant qu'il n'y a pas une histoire, mais des histoires de la psychanalyse, qui n'est donc pas elle-même un " sujet" homogène et autonome, préexistant au mouvement dans lequel il est entrainé, puisqu'elle se constitue au fur et à mesure qu'elle se modifie. A partir de là, il est possible de reposer, à la lumière de faits nouveaux, une question traditionnelle : qu'est-ce qui, en dehors de toute considération de légitimité et de garantie, fonde l'unité d'un savoir?

Il est impossible de reprendre le contenu des deux tomes de cet ouvrage d'E. Roudinesco, qui apportent, outre les éléments de réflexion qui viennent d'être suggérés, une immense masse d'informations. Dans le premier, on remarquera particulièrement la résurrection, extrêmement frappante, des deux figures de Charcot et de Pichon. Le second est évidemment dominé par l'aventure personnelle de Lacan, qui est ici restituée avec un grand effort d'objectivité, au-delà du débat, qui paraît aujourd'hui dérisoire, du "lacanisme " et de l" " antilacanisme". Ce qu'a entrepris E. Roudinesco, et ceci a naturellement pu paraitre scandaleux, c'est de montrer que la pensée de Lacan, au rebours de la forme dans laquelle elle a tendu elle-même à s'imposer, est inséparable de ses origines qui l'expliquent sans la réduire : le re-commencement de la psychanalyse, dans la mesure justement où il a été opéré dans les termes d'une rupture radicale, coïncide avec un «bricolage étonnant» (t. II, p. 413). E. Roudinesco écrit : « Le génie de Lacan ne ressemble guère à celui de Freud. Il réside moins dans la capacité de forger de toutes pièces un savoir nouveau que dans celle de rassembler, en un subtil bricolage, l'essentiel du savoir d'une époque " (t. II, p. 133). En effet, l'entreprise de Lacan a surtout consisté en une refonte de la théorie psychanalytique dans des termes qui lui étaient au départ étrangers, ceux de la philosophie, de l'anthropologie, de la linguistique, et pour finir de la logique mathématique : or, ce faisant, Lacan se trouve aussi avoir esquissé, d'une manière exemplaire, une synthèse de la culture de son temps. Vue dans l'œil de la psychanalyse, la pensée française se recompose en ses grandes lignes, en rejetant la fiction de son unité déjà donnée a priori : elle aussi, elle apparaît comme étant de façon permanente en travail. 